Sct. Morik. Diefe berifhmte Ruelle liegt in bem kohen Pergthale deb faínen Dberengabing. Die Baffers fpenoe in 1 Plinute 225,23 Rubizzoll. Nad v. $\mathfrak{B}$ u if bie Şshe beim unteren Flugi $557^{\prime}$, bei ber Brifa nad bem Sauerbrunnen 5391' und bie Rerdengránje nad oer Sirspeite $6985^{\prime}$ ũber ber Meereshibhe.

SФuls. Sn ber Flíde einer Duabratmeile finben fí bier 13 Säuerlinge, 1 Sdwefelquelle, „. Saljquellen. Fin frudtbares Bergthal, 3731' F̧auptquelle ift ber Steilling.

Tarap 8 liegt nur eine Stmndevon $\mathcal{S} d u[$ und $F$ is beris, in einem Pebenthale bes Эrettigatis, 3300 über ber JReeresidbe; Sct. $\mathfrak{B}$ ernharbin, in einem anmutbis geu Thale am füotiden Mabange bes Sct. Bernharbin; Syeiden, eine halbe Stunde vom Dorfe Eumbels.

Belb, eiue Therme, welde all ber weftliden $\mathfrak{B}_{\mathrm{erg}}$ feite zwifden Ect. Peter uno Taraps im Balertbale ents fpringt; bei $14^{\circ} \Re$. ber $\Re$ trmofpháre ift ibre Temp. $20^{\circ}, 5 \Re$.

Belvebere. Diefer Sduerling entquillt am fübsfts

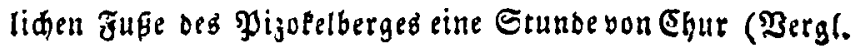
Allg. med. Annal, v. Pierer. 1827. Jan.).

Rügen's metallische Denkmäler der Vorzeit, vorzu gsweise chemisch bearbeitet und als Beitrag zur vaterländischen Alterthumskunde heransgegeben vom Prof. Dr. Hünefeld und Ferd. Picht. Mit Abbildungen. Leipzig 1827 . Verlag von L. Vols. gr. 8. S. VIII. u. 52 .

Diefe lleine, aber gebaltodle Ërift, foliegt fïh tout, dig an bie 2tbbanblungen úber vernantte Gigenftảne 
yon Sage, Rlaprotb, Dsbereiner, Burger u. a. Ebemifern. Eine groje Zahl ron ben Dentmalern bed alters thums ift in neuen Beiten nuf bem bertiden Fügen alts gegeben worben, inbbefonbere bat man manderlei metallis fhe Bexåthe aufgefunben. Es ift einleudtenb, bas bie

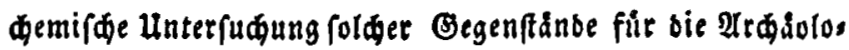
gie forobl, als soie für bie Ebernie ein bebeutenbed Ins tereffe bat. WBir lernen baburd bie Sompofitionen ber $\mathfrak{M}_{e=}$ tallmaffen genauer tenuen, welde bie Alten bearbeiteten unb lunnen mehr ober minder bebeutenbe Blide in bas

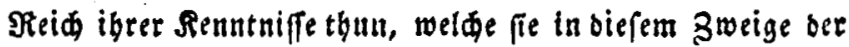
פBiffenf(aften uns Rinfte befapen "). Nigen ift befonders

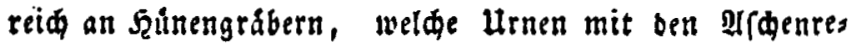
ffen ber Leiden, 2Baffen unb Bierrathen einfditepen. Die aubgebrannten snoden, welde bie urnen enthalten, befteben aus phobphorfaurem Ralf, mit einigen פorocenten toblenfauren Salf, naw Se y ff a $\mathrm{r}$ th, sie ddwarze Raffe ber Utrnen ift nad biefem Gielehrten ungetrocenet leidt zer, ftorbar, wirb in ber 2 Bdrme aber bart, unb foll nad Seyffartb altb 20 Sanb, 6o Riefel, 12,5 Ilauns erbe unb 7,5 Eifenozyd beftefien. Urnen faliefen foll, welde im Denabriafiden unb in sips piføen uno nameutlid in ber $\Re$ ahe von Saljuflen aubgegras ben fino, fo läft fíb bie 3ufammenfergung ber Saffe nut unvollfommen beftimnen; benn fie zeigt leine Şomogenität, fonbern beftebt alls einer thonigen Rafle, in welde viele Partieln von Sand unb anbern Steinden eingefittet ind. Sndeffen iff es gewis, daßj die Urnen in ber Erbe febe weid finb unb nur mit groper Sorgfalt ausgegraben wets

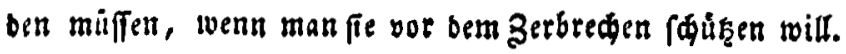

•) S. in meinem Repertor. fúr die Ehemie D. II. ben Att. Antiquitaten. $B$ r. 
In ber \&uft wirs bie Maffe balo hart. Deim Afusgraben liepin wir bier bet llflen die Erbe umber furgfilltig wegs nchmen unb die entbroffen hrnen einige Tage rubig auf ibrer Etelleftelyen, fie waren albandm fo weit erbirter, oaj fie ohne Sejurgnis weggenommen werden Eunntell, abgebrodule

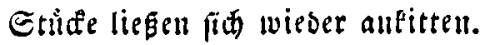

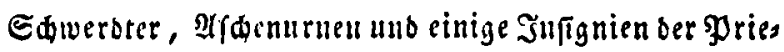
fler fittb bie wejentliditen Geráthe, sie mall finbet. Sdimuffachen und metallifheb $\mathfrak{S}_{2}$ auggerith findet man

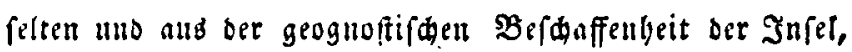
bie wefentlic aud Granit, Freibe und Ibonlagen beftebt,

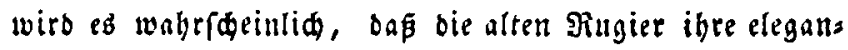
teren uruen und metallifden Beratbe von alidern Stám men ober voll ben Sismern bejogen.

Das̆ Rupfer bvat bei ben Altten ber Stellyertreter bes Eifeno, weldes man erft fpáter fennell lernte. Die Egys ptier faunten jwar fdon 1600 Sabre vor Ehrifto ben Stabl, femer fpridht vom fparfamen Bebrall does ouspops, die Rumer hatten 300 nor Ebrifto nurifdes Eifen; die eigent: lide $\mathfrak{B e a r b e i t u n g ~ D i e f e b ~ M e t a l l b ~ g e f d a l s ~ a b e r ~ e r f t ~ n a d ~}$ orm Mittelalter. Die eifernen Denfmaler des Alterthums rübren baber aแs beแ fpáteren 3eiten bes Einfalls ber RSmer ber. Das Rupfer verfegten bie alten mit Zinn, rooburd

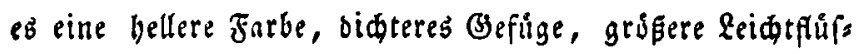
figleit, Eprobigleit, Şâtte unb Rlang erbilt. Die Sprs,

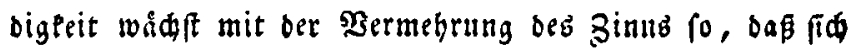

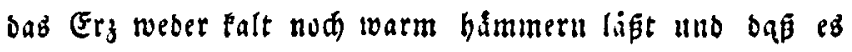
wahrfdeinlid wird, daß bie meiften antiquarifhen Gegens fande biefer Sompofitionen fenn gegoffen worben, uno daf nur die gebammert twarben, bei benen bas Silipfer libers miegcto war.

Fin metallifdea Edwerbt ober Spfermeffer beftano aus a sinn auf 5,6 Rupfer. Die Alualyfe fieltrn bie $\mathfrak{B}$. 
an, inbem fie bas Metall mit Galpeterfaure codtent unb aus bem gebildeten uno gefammelten Bimnoxpbe bas 3inn beredneten, unb barauf bie falpeterfaure supferallfisfung vorfidtig abraudten, ben Rlifftand glibeten unb aub bem erbaltenen fimarzen Supferoxpde bas Sitpfer berednes ten. Soll lę̧tere Beftimmung genalle Refultate geben,

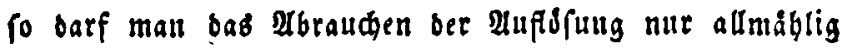
yollenben, weif bas Salpetergab leidt Rupferosyo mit aufreift. Es ift beshalb gut, einen weithaliigen Porcellantrid,

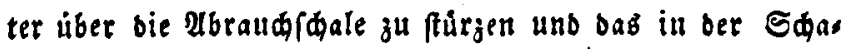

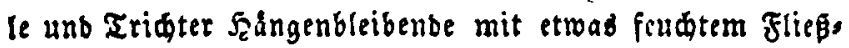
papier in ben Tiegel zu bringen, uno biefes mit ju verbren, nen, nadbem man zuvor ben 21/dengeḩalt bes şapiers Fennt, unb endid ise Brůgung eine Etunde lang fortjus

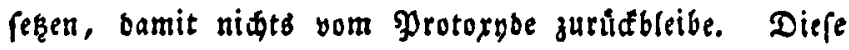
Methobe zieben bie $\mathfrak{B}$. ben ubrigen yor.

Fine metallifae Urme: Der Roft derfelben beftanb alls toglenfaurem Supferosyb, Sinnosys uns Rupferoxybul. Die Eompofition ber Urme war 90,33 Supfer alf 9,67 Binn, alfo ber SMiffung unferes ję̧igen Sanonenmetalls analog. Wenn lez̧te Mifdung langfam ertaltet, to f由is, bet fid ba Sinn yom Sipper $a b$ und man fintet alf ber Brufflade bes verlorenen Sopfi ber fanounen nad bem Biefen eine medanilde Mengung von 3inn und/Supfer uno unter anbern eine pordfe Maffe yon weniger zinmhals tigem Supfer; Berbaltniffe, welde aud an biefer urme yortamen. Fin metallifwer Ring zeigte eine faft gleide Eompofition wie bie urne. Ein anberes Edwerbt, ein Streitmeisel unb ein Dold, waren in ibren Sompofitionen mit ben zuerft unterfudten faft ibentifक. Die Spiz̧e cinet Lanje enthielt auf 7,3 Rupfer 1 3iun. Ein mefiñnartiges Flafdien beftand aus Supfer uno zint. Die hohlen frin, ranbigen Ringe, bie fid in eiuer fogenannten Damenurne 
bejanben, entbielten altf 92 Siupfer nut 8 şinn; biefe Eompofition war alfo bie fupferbaltigfte, unb barallb ers Hart ed fid, warum bic Ilten to binnwandige Saden bars aus arbeiten fonnten. Fine grjpere, ein gefdlungener Ring, beftanb alts 65 flupfer uno 31 Silber, eine Sompofition, bie fid nidat gut balt unb an ber \&uft fdwar anlafuf.

Sn einem \$nhang berihgren die Serf. mebrere nidt uns widrtige Bregenftánde, yon benen wir nod Finiges mittheis Ien. Berjeli lls bemerft in feinem \&ehrbude, bas bas Durd Ealpeterfäure bargeftellte Bimespo in Salpeterfaure

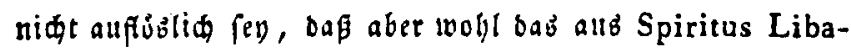
vii bargeftelle forvohl in Edwefel: uno Ealzfäure, alb alt zu geringen Theilen in Salpeterfáre fid aufiofe. Die Berf. fanben mun jwar bei ifren linterfudungen, baß bes fonders bei Eleinen Mengen ber fegirung bie falpeterfaure Rupfer : 2luflsfung teine bemerfbaren Mengen yon Bimnoryb beim $\mathfrak{B e r b a m p f e n ~ z u r i f t i e f , ~ w e n n ~ a b e r ~ b i e ~ e r b a l t e n e n ~} \mathbb{P u}=$ pferojybe julammengenommen in Salpeterfinte aufgeldit unb bie alufsfung verdunftet wurbe, fo fibieb fich bod etwas ainnorvenl alts; fo baf mithindie Salpeterfánee, wenn fie firt jeue Eompopitionen aud bas befre allalytifae Nittel ift, gegen bas হaiun, unlsatideg D,yobitbento, niđt fo ins bifferent a feyn fdeint, als maninggemein glubbe, was and beim 2 utimon ill nod bübrem Grabe vorpsumt. Nad bell

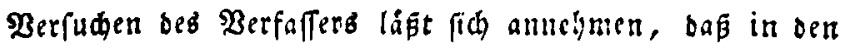
obigen Berbiltniffen gegen 100 Rupfer nud 2,84 3inn fíd finber. Diefe Eorrection iff bei ben yorftehenben Shalyfen in Redanng gebradt worben. Die Urfade, warumbit biefen Berfudenzinnoryb aufgeluff seirb, fuden bie Betf. darin, bag

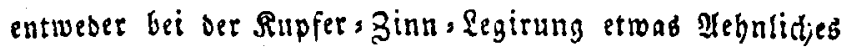
Statt finoet, wie beim filberhaltigen Golbe, unb bei ben legirıngen, wo dab eine Metall bab anbere fautst ober in

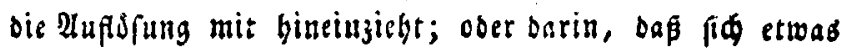


Binnosysulbitbe, ober enblid am magr foeinfiaften barin, ba fid bei ber Bilbung des falpeterfauren Ümmoniaps, welded je: besınal entfteht, wenn aiinn in Salpeterfäure aufgelsft wiro"), ei uauflustides Doppelfal $l_{3}$ erjeugen; móglida aud, onß bieallfs lojung bes 3innorydes von ber beim 2lbrallden entjęhenden falpetrigen Salure berribren tanu.

Fermer bemerlten bie \$erfaffer, baß bie Nufufung you Sulphas cupricus bei ber Digeftion eine grofe Menge Rlls pieroryb alf:ah und damit einen weiglidgrinen Riebers

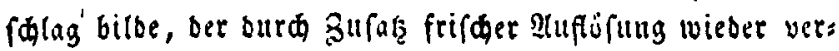
forwinbet. Diefe Pluflúfung giebt dann Siryftalle, welde eime bellere blaue Farbe baben aló bas Sulphas.

Die Rigenfden Alterthimer jeigten eben fo, wie $\Im$. Dauy, Nuggeratb uno Mubere eb bei águliden (jes genffinben bemerften, Uleberjige von Rupfers und Rupfers oxybulfryftalle. Die Berfaffer fino oer Meinumg, baß biefe Biroung eine Folge bes Guffes diefer Berathe fey, da man halufig findet, dab beim Erialteu der gefdmolzenen \&egis rungen Eryftallinifie Ilusfonberungen entfteben. Sou bies fer $\mathfrak{A}$ rt finb nun wabrfdecinfid jene Sryltalle ber Mllterthí: ner, welde nod in ber legirung frzen, unb ben Finwirs tungen mebre als biefe felbft wiberfeben fontten, eben ibs ret fryfallinifon Bilbung wegen. Sie wurben fellens weife freigclegt, und frellenweife von $\$$ xyo umgeben. Nit biefen Rupferfryftallen fdeinen Mebrere bns Drubul vers wedfelt ju haben, wab wohl nut baburd eine eryffallinis fde Struftur getoonnen batte, dá jene Supfertryftalle alt einigen Stelfen loeniger gefdîht, ober ftárterer Eintoirtuns gen altzgefefzt, oxyoulitt wurben unb fo bem Dyoulle sine

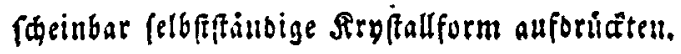

*) Detgl. ben $\mathfrak{A r t}$. Immoniaf im etfen $\mathfrak{3}$ ande meined Ples pert. fút die Ebemie, vei $\mathfrak{B}$ ilbung bes Ammoniats. $\mathfrak{B}$ r. 
Diefe Sdrift hat alfo porobl bie wemifhe Arterthumbs Punbe bereidert, als aud unfere demifden Renntniffe in mebreren Yunten ermeitert unb beridtigt, und verbient bie

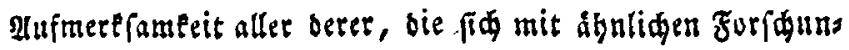
gen abgeben. $\mathfrak{B} r$.

\section{Sapitain $\mathfrak{\Omega}$ ter $\mathfrak{z}$ Shgrometer *).}

Diefes befteht alls einem Duedfilberthermometer, beffen grabuirte Ecale obngefabr $4 \frac{\mathrm{r}}{2}$ 3oll lang iff, ain untern Ende ber Scale ift bie Rshre in Form eines redten Mintels ges bogell, all beffen Ende bie Rugel parallet ohngefăbr 1 3oll yon ber Rolgre entferint auffeigt; die Rugel if ohngefiht einen 3oll lang, crlindrif Epiţe, beren Durdmeffer etwas grsper ift, als bie seb cylinbrifden mit Seide umgebenen হheils. Die Gale ift an einen cylindrifhen Dratb befeftigt, welder 5 zoll lang ift unb in ein Edranbengerwinbe fajt. Daballbere Enbe bies fes Drath́, weldes als x:áger bes Suftruments sient, ift in einem Fufgeftell befeftigt, weldes auf ber Innenceite an einem Enbe bes Raftens fï befindet. Der Saffenenthilt zugleid eill fleines (3lab fủr 2lether. Das to couftruite Thermometer giebt bie Temperatur ber Ruft uno sen Tualls puntet an, wenn man auf bem bebecten Theil ber Sugel Ietber tripfelt, welder barna abfieft, olyne die Spiţe ber Sugel ju ndffen, welde fogleid burd bie barauf fid nies berfalagende Fendtigeit der \{uft eine matte Sbers flide befommt, woranf man die beobadtete Temperatur uns bie Differenz yon ber ber\{uft beftimmt. Fin ăntides Juftrus ment bat $\mathfrak{Y}$ rofeffor $\mathfrak{B} a \mathfrak{m g a r}$ ten in $\mathfrak{B}$ ien angegeben.

*) Philos. Transact. London 1326. Annals of Philos. N. S. XII. 52. 